PROCEEDINGS OF THE

AMERICAN MATHEMATICAL SOCIETY

Volume 137, Number 12, December 2009, Pages 4099-4107

S 0002-9939(09)09988-2

Article electronically published on July 15, 2009

\title{
ISOLATED SINGULARITIES FOR THE EXPONENTIAL TYPE SEMILINEAR ELLIPTIC EQUATION IN $\mathbb{R}^{2}$
}

\author{
R. DHANYA, J. GIACOMONI, AND S. PRASHANTH
}

(Communicated by Matthew J. Gursky)

\begin{abstract}
In this article we study positive solutions of the equation $-\Delta u=$ $f(u)$ in a punctured domain $\Omega^{\prime}=\Omega \backslash\{0\}$ in $\mathbb{R}^{2}$ and show sharp conditions on the nonlinearity $f(t)$ that enables us to extend such a solution to the whole domain $\Omega$ and also preserve its regularity. We also show, using the framework of bifurcation theory, the existence of at least two solutions for certain classes of exponential type nonlinearities.
\end{abstract}

\section{INTRODUCTION}

Let $\Omega \subset \mathbb{R}^{2}$ be a bounded domain with $0 \in \Omega$. Denote $\Omega^{\prime}=\Omega \backslash\{0\}$. Let $f:(0, \infty) \longrightarrow(0, \infty)$ be a locally Hölder continuous function which is nondecreasing for all large $t>0$. In this article we study the following problem:

$$
\left\{\begin{aligned}
-\Delta u & =f(u) \\
u & \geq 0 \\
u & \in L_{l o c}^{\infty}\left(\Omega^{\prime}\right) .
\end{aligned} \text { in } \Omega^{\prime},\right.
$$

It is well-known from the works of Brezis-Lions [5] that if $u$ solves $\left(P^{\prime}\right)$, then indeed $u$ solves the following problem in the distributional sense in the whole domain $\Omega$ :

$$
\left\{\begin{aligned}
-\Delta u & =f(u)+\alpha \delta_{0} \\
u & \geq 0 \\
\alpha \geq 0, u, f(u) \in L_{l o c}^{\infty}\left(\Omega^{\prime}\right) \cap L_{l o c}^{1}(\Omega) . & \text { in } \Omega,
\end{aligned}\right.
$$

This leads us to the following two questions:

(Q1) Can we find a sharp condition on $f$ that determines whether or not $\alpha=0$ in $\left(P_{\alpha}\right)$ ?

(Q2) If $\alpha=0$, is it true that $u$ is regular (say, $C^{2}$ ) in $\Omega$ ?

We make the following

Definition 1.1. We say $f$ is a sub-exponential type function if

$$
\lim _{t \rightarrow \infty} f(t) e^{-\beta t} \leq C \quad \text { for } \text { some } \beta, \mathrm{C}>0 .
$$

We say $f$ is of super-exponential type if it is not a sub-exponential type function.

Received by the editors September 30, 2008.

2000 Mathematics Subject Classification. Primary 35B32, 35B65, 35J25, 35J60.

Key words and phrases. Isolated singularity, blow-up, Laplace equation.

(C)2009 American Mathematical Society 
As a complete answer to question (Q1) we show (Theorem 2.1) that if $f$ is of super-exponential type, then $\alpha=0$, and conversely (Theorem 2.2) that $\left(P_{\alpha}\right)$ has solutions for small $\alpha>0$ if $f$ is of sub-exponential type.

Similarly, we answer question (Q2) by showing that for any $f$ of sub-exponential type, any solution $u$ of $\left(P_{0}\right)$ is regular $\left(C^{2}\right)$ inside $\Omega$ (Theorem 3.1). Conversely, for $f$ of super-exponential type with any prescribed growth at $\infty$ and behaviour for small $t>0$, in Lemma 3.1 and Theorem 3.3 we construct solutions $u$ of $\left(P_{0}\right)$ that blow-up only at the origin. To our knowledge, the existence of such singular solutions has not been considered so far for super-exponential type problems. Theorem 3.2 should be contrasted with the results in [2] and [13. Particularly in [13, the nonlinearity under study is of a model type, viz., $f(t)=e^{t^{\mu}}, \mu>0$. These authors show that for a noncompact sequence of solutions to $\left(P_{0}\right)$ posed on a ball, concentration phenomenon occurs for $1<\mu<2$ and total blow-up occurs for $\mu<1$. Clearly, $\mu=1$ appears as the borderline exponent between total blow up and concentration. In Theorem 3.2, when $\mu=2$, for certain classes of nonmodel type nonlinearities we show that instead of concentration, convergence to a singular solution occurs. If the nonlinearity is closer to a model-type, more precisely, if $\liminf _{t \rightarrow+\infty} f(t) e^{-t^{2}} t=+\infty$, then only concentration takes place, as follows from the results in [1].

Definition 1.2. We denote by $\Gamma$ the fundamental solution of $-\Delta$ in $\mathbb{R}^{2}$. That is, $\Gamma(x)=-\frac{1}{2 \pi} \log |x|, x \in \mathbb{R}^{2} \backslash\{0\}$.

\section{Extendability of the SOlution From the PUnCtURed DOMAin TO THE ENTIRE DOMAIN}

In this section, we will discuss the extension of a solution of $\left(P^{\prime}\right)$ to the whole domain $\Omega$.

Theorem 2.1 (Removable singularity). Let $f$ be of super-exponential type. Then any solution $u$ of $\left(P^{\prime}\right)$ extends to a distributional solution of $\left(P_{0}\right)$.

Proof. As noted before for some $\alpha \geq 0, u$ solves $\left(P_{\alpha}\right)$. Therefore, $-\Delta(u-f(u) *$ $\Gamma-\alpha \Gamma)=0$. Since $f(u) * \Gamma \geq 0$ it follows that $u(x) \geq \alpha \Gamma(x)-C$ for all $x \in \Omega$ for some constant $C>0$. Since $f(t)$ is nondecreasing for all large $t>0$, we obtain, for any $\delta>0$ small enough, $f(\alpha \Gamma(x)-C) \leq f(u(x))$ for all $|x|<\delta$. If $\alpha>0$, we choose $\beta=\frac{4 \pi}{\alpha}$ and apply Definition 1.1 to obtain $|x|^{-2} \leq f(u(x))$ for all $|x|$ small. This contradicts the fact that $f(u) \in L_{l o c}^{1}(\Omega)$. Hence, necessarily, $\alpha=0$.

Define

$$
\beta^{*}=\inf \{\beta>0 \text { occurring in Definition } 1.1\} .
$$

Then, we can show the following:

Theorem 2.2. Let $f$ be a sub-exponential type nonlinearity. Then for all $\alpha \in$ $\left(0, \frac{2 \pi}{\beta^{*}}\right)$ the problem $\left(P_{\alpha}\right)$ admits a solution. Furthermore, if $f(t) \geq C e^{\bar{\beta} t}, \forall t \geq 0$, for some $\bar{\beta}>0$, then $\left(P_{\alpha}\right)$ has no solution for all $\alpha \geq 4 \pi(\bar{\beta})^{-1}$.

Proof. We will prove the above statements using the monotone iteration technique. In fact, we will construct a solution $u$ of $\left(P_{\alpha}\right)$ which vanishes on $\partial \Omega$ for all suitable $\alpha$. Without loss of generality, for this purpose we assume that $f$ is nondecreasing for all $t>0$ (if not, we replace $f(t)$ by $f(t)+k t$ for some large $k$, use the argument below and recover the result for $f)$. It is clear that $u_{0}=0$ is a sub-solution of $\left(P_{\alpha}\right)$ for all $\alpha>0$. 
Let us define, for any $\beta, C>0$ given by Definition 1.1

$$
v_{\beta}(x)=-\beta^{-1} \log \left(4|x|\left(1+\frac{\beta C}{4}|x|\right)^{2}\right) .
$$

Then a simple computation gives $-\Delta v_{\beta}=2 \pi \beta^{-1} \delta_{0}+g$, where

$$
g(x)=C\left(2|x|\left(1+\frac{\beta C}{4}|x|\right)^{2}\right)^{-1} .
$$

It can be easily checked that $g \in L^{r}(\Omega)$ for all $1<r<2$ and $g \geq f\left(v_{\beta}\right)$ in $\Omega$. Hence $v_{\beta}$ is a supersolution of $\left(P_{\alpha}\right)$ for all $\alpha \leq 2 \pi \beta^{-1}$. Now, for any such $\alpha$, consider the following sequence of problems:

$$
\left\{\begin{array}{ccccc}
-\Delta u_{n} & =f\left(u_{n-1}\right)+\alpha \delta_{0} & & \text { in } & \Omega, \\
u_{n} & =0 & & \text { on } & \partial \Omega, \\
u_{n} & \in L^{p}(\Omega) & & 1<p<\infty, \\
f\left(u_{n}\right) & \in L^{r}(\Omega) & & 1<r<2 .
\end{array}\right.
$$

We construct a solution $u_{n}$ of the problem $\left(P_{n}\right)$ inductively as follows. Let $w_{1} \in$ $C^{2}(\bar{\Omega})$ be the solution of the problem

$$
\left\{\begin{array}{ccccc}
-\Delta w_{1} & = & f\left(u_{0}\right) & \text { in } & \Omega, \\
w_{1} & = & -\alpha \Gamma & \text { on } & \partial \Omega .
\end{array}\right.
$$

Define $u_{1}=w_{1}+\alpha \Gamma$. It can be easily seen that $u_{1}$ is a solution for $\left(P_{1}\right)$ with $f\left(u_{1}\right) \in L^{r}(\Omega)$ for $1<r<2$. Now assume that there exists a solution for $\left(P_{n-1}\right)$. Let $w_{n} \in W^{2, r}(\Omega)$ be a solution of

$$
\left\{\begin{array}{ccccc}
-\Delta w_{n} & = & f\left(u_{n-1}\right) & \text { in } & \Omega, \\
w_{n} & = & -\alpha \Gamma & \text { on } & \partial \Omega .
\end{array}\right.
$$

By standard elliptic regularity $w_{n}$ is a Hölder continuous function in $\bar{\Omega}$. Then $u_{n}=$ $w_{n}+\alpha \Gamma$ solves $-\Delta u_{n}=f\left(u_{n-1}\right)+\alpha \delta_{0}$ in $\Omega$, and $u_{n}=0$ on $\partial \Omega$. Also $u_{n} \in L^{p}(\Omega)$ for every $1 \leq p<\infty$. Next we notice that $u_{n}-v_{\beta}$ solves $-\Delta\left(u_{n}-v_{\beta}\right) \leq f\left(u_{n-1}\right)-g$ a.e. in $\Omega$ and $u_{n}-v_{\beta} \leq 0$ on $\partial \Omega$ (for $C$ large enough). Hence by the maximum principle $u_{n} \leq v_{\beta}$ in $\Omega$. Also we notice that $f\left(v_{\beta}\right) \in L^{r}(\Omega)$ for $1 \leq r<2$. Using the monotonicity of $f$ we conclude that $f\left(u_{n}\right) \in L^{r}(\Omega)$ for $1 \leq r<2$. Hence we have obtained a sequence $\left\{u_{n}\right\}$ solving $\left(P_{n}\right)$ and

$$
u_{n} \leq v_{\beta} \quad \text { in } \Omega \quad \text { for all } n \in \mathbb{N} .
$$

It can also be shown easily that $0 \leq u_{1} \leq u_{2} \cdots \leq u_{n-1} \leq u_{n} \cdots$. Now define $u(x)=\lim _{n \rightarrow \infty} u_{n}(x)$. Then it follows that $u$ is a solution to the problem $\left(P_{\alpha}\right)$ for any $\alpha \leq 2 \pi(\beta)^{-1}$. Since $\beta \geq \beta^{*}$ we indeed have a solution to $\left(P_{\alpha}\right)$ for all $\alpha<2 \pi\left(\beta^{*}\right)^{-1}$.

Let us now take $f(t) \geq C e^{\bar{\beta} t}, \forall t \geq 0$, for some $\bar{\beta}>0$. Suppose there exists a solution $u$ of $\left(P_{\alpha}\right)$. We then have $u \geq-\frac{\alpha}{2 \pi} \log |x|-C_{1}$ in $\Omega$, which, if $\alpha \geq \frac{4 \pi}{\bar{\beta}}$, contradicts the basic conclusion that $f(u) \in L_{l o c}^{1}(\Omega)$.

We then have the following:

Corollary 2.1. If $f(t) \leq C e^{\beta t^{\mu}}, \forall t \geq 0$, for some $0<\mu<1$, then $\beta^{*}=0$, and hence $\left(P_{\alpha}\right)$ admits a solution for every $\alpha>0$. 


\section{Regularity AND THE LACK of IT FOR THE EXTENDABle SOLUTiON}

In this section we discuss question (Q2) and show that regularity or the lack of it for the solution to $\left(P_{0}\right)$ is determined by whether $f$ is of sub-exponential type. As an application of results in Brezis-Merle [6] we have the following:

Theorem 3.1. Let $f$ be a sub-exponential type nonlinearity. Then any solution $u$ of the problem $\left(P_{0}\right)$ is regular in $\Omega$.

Proof. Let $u$ solve $\left(P_{0}\right)$. By Corollary 5.2 in [6], $e^{k|u|} \in L^{1}(\Omega)$ for every $k>0$. Therefore, since $f$ is of sub-exponential type, we obtain that $f(u) \in L^{r}(\Omega)$ for every $1<r<\infty$. Let $u_{1}$ be the solution of

$$
\left\{\begin{array}{cccc}
-\Delta u_{1} & = & f(u) & \text { in } \Omega, \\
u_{1} & = & 0 & \text { on } \partial \Omega .
\end{array}\right.
$$

Then, $u_{1} \in W^{2, r}(\Omega)$ for all $r>1$. Therefore, by Sobolev embedding $u_{1} \in C^{1, \theta}(\bar{\Omega})$ for every $0<\theta<1$. But in the interior of $\Omega$ we have, in the sense of distributions, $\Delta\left(u-u_{1}\right)=0$. Then, it is well-known that $u=u_{1}+h$ a.e. for some harmonic function $h$. Therefore, $u$ is Hölder continuous in $\Omega$, and by standard elliptic regularity, it is $C^{2}$ inside $\Omega$.

In the next two proofs we construct solutions of $\left(P_{0}\right)$ which blow-up at the origin when $f$ is of super-exponential type. Let $B_{R}$ denote the open ball of radius $R$ centered at the origin.

Lemma 3.1. Given any $\mu>1$ there exists an $f$ of super-exponential type satisfying $\lim _{t \rightarrow \infty} f(t) e^{-t^{\mu}}=0$ such that the corresponding problem $\left(P_{0}\right)$ posed on the unit ball $B_{1}$ admits a solution that blows-up at the origin.

Proof. Given $\mu>1$, define $f(t)=4(\mu-1) \mu^{-2} t^{1-2 \mu} e^{t^{\mu}}, t>0$. Clearly $f$ satisfies the requirements stated in the lemma. It can be checked that if we define $u(x)=$ $(-2|\log | x||)^{\frac{1}{\mu}}, x \in B_{1}$, then, thanks to Theorem 2.1] $u$ solves $\left(P_{0}\right)$ with the above choice of $f$.

In the above result, though we could choose $f$ satisfying any prescribed superexponential type growth at infinity, the behaviour for small $t>0$ is of singular type. In the next result we exhibit super-exponential type nonlinearities whose growth rate at infinity is fixed (in fact it grows like $e^{t^{2}}$ as $t \rightarrow \infty$ ) but has regular behaviour for small $t>0$. For this we need to use the nonexistence results proved in [3], which together with Theorem 3.3 stated below helps us to show the following:

Theorem 3.2. Let $f:[0, \infty) \rightarrow[0, \infty)$ be a $C^{3}$ super-exponential type nonlinearity with $f(0)=0$, which has the form $f(t)=h(t) e^{t^{2}}$, where $h(t)=e^{-t^{\mu}}|\log t|^{p}, \mu \in$ $(0,2), p \geq 0$, or $h(t)=t^{-\theta}, \theta \geq 1$, for all large $t>0$. Then there exists $R_{*}>0$ such that $\left(P_{0}\right)$ posed on $B_{R_{*}}$ admits a radial solution blowing up at the origin.

Proof. We first assume the proof of Theorem 3.3. Then the nonexistence results contained in Theorems A and B of [3] imply that the assumptions of Theorem 3.3 hold and therefore the existence of a radial solution blowing-up at the origin.

Consider the following problem which is a regular version of $\left(P_{0}\right)$ posed on $B_{R}$ :

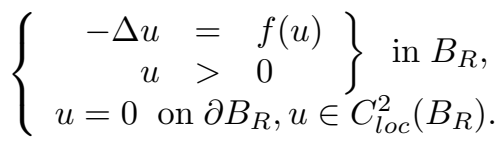


Theorem 3.3. Let $f:[0, \infty) \rightarrow[0, \infty)$ be a $C^{3}$ super-exponential type nonlinearity such that $g \triangleq \log f$ is convex for all large $t>0$. Suppose there exist a sequence $\left\{R_{n}\right\}$ of positive real numbers with $R_{*} \triangleq \liminf _{n \rightarrow \infty} R_{n}>0$ and a sequence $\left\{u_{n}\right\}$ of solutions to $\left(P_{R_{n}}\right)$ such that $\sup _{B_{R_{n}}} u_{n} \rightarrow \infty$ as $n \rightarrow \infty$. Then the problem $\left(P_{0}\right)$ posed on $B_{R_{*}}$ admits a solution that blows up only at the origin.

Proof. In order to prove the theorem, we perform some transformations that will put $\left(P_{R}\right)$ into the equivalent form of the classical Emden-Fowler equations. First, we observe that thanks to the symmetry result of Gidas-Ni-Nirenberg, any solution of $\left(P_{R}\right)$ is radially symmetric and, in fact, strictly radially decreasing about the origin. Therefore, $\left(P_{R}\right)$ can be rewritten as the following ODE boundary value problem via the transformation $w(r)=u(|x|=r)$ for $r \in(0, R)$ :

$$
\left\{\begin{array}{c}
-\left(r w^{\prime}\right)^{\prime}=r f(w) \\
w>0 \\
w^{\prime}(0)=w(R)=0 .
\end{array}\right\} \text { in }(0, R),
$$

We finally make the following Emden-Fowler transformation:

$$
y(t)=w(r), \text { where } r=2 e^{-\frac{t}{2}}, \quad t \in\left(2 \log \left(2 R^{-1}\right), \infty\right) .
$$

Then it can be checked that $\left(P_{R}\right)$ is equivalent to the following problem with $T=$ $2 \log \left(\frac{2}{R}\right)$ :

$$
\left.\begin{array}{rl}
-y^{\prime \prime} & =e^{-t} f(y) \\
y & >0 \\
& y(T)=y^{\prime}(\infty)=0 .
\end{array}\right\} \text { in }(T, \infty),
$$

For our purpose, instead of the above boundary value problem, it will be more convenient to consider the following initial-value problem depending upon a parameter $\gamma>0$ :

$$
\left\{\begin{array}{c}
-y^{\prime \prime}=e^{-t} f(y) \\
y(\infty)=\gamma, y^{\prime}(\infty)=0
\end{array}\right.
$$

Since $f(y(t))>0$ as long as $y(t)>0$, it follows from $\left(P_{\gamma}\right)$ that $y$ is a strictly concave function as long as it is positive. Therefore, there exists $T_{0}(\gamma)>-\infty$ such that $y\left(T_{0}(\gamma)\right)=0$ and $y(t)>0$ for all $t>T_{0}(\gamma) . T_{0}(\gamma)$ thus defined is clearly the first zero of the solution $y$ of $\left(P_{\gamma}\right)$ as we move left from infinity. Let $y_{0}>0$ be such that $g$ is convex for all $t>y_{0}$. We also define the point $t_{0}(\gamma)>T_{0}(\gamma)$ to be such that $y\left(t_{0}(\gamma)\right)=y_{0}$ for each $\gamma>0$.

Our idea is to obtain the blow-up solution of $\left(P_{0}\right)$ posed on $B_{R_{*}}$ as the upper envelope of the sequence of solutions $\left\{u_{n}\right\}$. Let $\gamma_{n}=u_{n}(0)$ and $\left\{y_{n}\right\}$ be the corresponding sequence of solutions to $\left(P_{\gamma_{n}}\right)$. Thanks to our assumptions it follows that $\gamma_{n} \rightarrow \infty$ as $n \rightarrow \infty$ and $T^{*} \triangleq \limsup _{n \rightarrow \infty} T_{0}\left(\gamma_{n}\right)<\infty$ (we remark that $\liminf _{n \rightarrow \infty} T_{0}\left(\gamma_{n}\right)$ can be $\left.-\infty\right)$. By definition, $T^{*}>-\infty$. We make the following claim.

Claim. $\left\{y_{n}\right\}$ is a uniformly bounded sequence on compact subsets of $\left[T_{*}, \infty\right)$.

Proof of claim. We define the following energy function associated to $\left(P_{\gamma_{n}}\right)$ :

$$
E_{n}(t)=y_{n}^{\prime}-\frac{1}{2}\left(y_{n}^{\prime}\right)^{2} g^{\prime}\left(y_{n}\right)-e^{g\left(y_{n}\right)-t}, t \geq T_{0}\left(\gamma_{n}\right) .
$$


Hence, $E_{n}^{\prime}(t)=-\frac{1}{2}\left(y_{n}^{\prime}\right)^{3} g^{\prime \prime}\left(y_{n}\right) \leq 0, \forall t \geq t_{0}$, since $y_{n}$ is strictly increasing and $g$ is convex for this range of $t$. Since $\lim _{t \rightarrow \infty} E_{n}(t)=0$ we obtain that $E_{n}$ is a nonnegative function on $\left(t_{0}\left(\gamma_{n}\right), \infty\right)$. This immediately implies that

$$
y_{n}^{\prime}(t) g^{\prime}\left(y_{n}(t)\right)<2, \forall t \geq t_{0}\left(\gamma_{n}\right) .
$$

Now, integrating the ODE in $\left(P_{\gamma_{n}}\right)$ we have

$$
\int_{t_{0}\left(\gamma_{n}\right)}^{\infty} f\left(y_{n}(t)\right) e^{-t} d t=y_{n}^{\prime}\left(t_{0}\left(\gamma_{n}\right)\right) .
$$

From (3.1) and recalling that $y_{n}\left(t_{0}\left(\gamma_{n}\right)\right)=y_{0}$, we get

$$
\sup _{n} \int_{t_{0}\left(\gamma_{n}\right)}^{\infty} f\left(y_{n}(t)\right) e^{-t} d t<\infty .
$$

If now $t_{0}\left(\gamma_{n}\right) \rightarrow \infty$ as $n \rightarrow \infty$, then clearly the claim holds for any interval $[a, b] \subset\left[T_{*}, \infty\right)$. Suppose for some subsequence of $\left\{\gamma_{n}\right\}$, denoted again by $\left\{\gamma_{n}\right\}$ for convenience, we have $\lim \sup _{n \rightarrow \infty} t_{0}\left(\gamma_{n}\right)<\infty$. It is enough to show, in view of the monotonicity of $y_{n}$, that $\left\{y_{n}(t)\right\}$ is a bounded sequence of real numbers for any $t \in\left[T_{*}, \infty\right)$. If this is not true, then for some subsequence of $\left\{y_{n}(t)\right\}_{n \geq 1}$, we will have $\lim _{n \rightarrow \infty} y_{n}(t)=\infty$. Clearly, such a $t$ has to be larger than $t_{0}\left(\gamma_{n}\right)$ for all large $n$. In view of monotonicity of $y_{n}$ again, it follows that $y_{n} \rightarrow \infty$ uniformly on $[t, t+1]$, which contradicts (3.2). Thus we prove the claim in this case also.

Define

$$
y(t)=\sup _{n \geq 1} y_{n}(t), \quad t>T_{*} .
$$

Clearly, $y$ is positive and nondecreasing on $\left[T_{*}, \infty\right)$. For each $n$, choose $T_{1}\left(\gamma_{n}\right)>T_{*}$ by the rule $y_{n}\left(T_{1}\left(\gamma_{n}\right)\right)=\frac{\gamma_{n}}{2}$. Clearly, $T_{1}\left(\gamma_{n}\right) \rightarrow \infty$ as $n \rightarrow \infty$ and $y\left(T_{1}\left(\gamma_{n}\right)\right) \geq \frac{\gamma_{n}}{2}$. Hence $y\left(T_{1}\left(\gamma_{n}\right)\right) \rightarrow \infty$ as $n \rightarrow \infty$. By the monotonicity of $y$ we conclude that $y(t) \rightarrow \infty$ as $t \rightarrow \infty$. By Helly's theorem, up to a subsequence, $y_{n} \rightarrow y$ pointwise a.e. in $\left[T_{*}, \infty\right)$. Integrating the ODE satisfied by $y_{n}$ twice, we get

$$
y_{n}(t)-y_{n}(s)=\int_{s}^{t}(\rho-s) f\left(y_{n}(\rho)\right) e^{-\rho} d \rho, \quad T_{*}<s<t<\infty .
$$

Passing to the limit as $n \rightarrow \infty$ on either side of the above equation we obtain that $y$ also satisfies the same integral equation for a.e. $t$ in $\left(T_{*}, \infty\right)$. From (3.2) and Fatou's Lemma, we obtain that $\int_{T_{*}}^{\infty} f(y(t)) e^{-t} d t<\infty$. Thus, $y$ solves the differential equation $-y^{\prime \prime}=e^{-t} f(y)$ in $\left(T_{*}, \infty\right)$ with $\int_{T_{*}}^{\infty} f(y(t)) e^{-t} d t<\infty$. Going back to our original variable $x \in B_{R_{*}}$ and defining $u(x)=y\left(2 \log \left(\frac{2}{|x|}\right)\right)$, we obtain that $u$ solves the following problem:

$$
\left\{\begin{array}{c}
-\Delta u=f(u) \\
u>0 \\
\lim _{|x| \rightarrow 0} u(x)=\infty \\
\int_{B_{R_{*}}} f(u)<\infty
\end{array}\right.
$$

By the result of Brezis and Lions [5], in fact $u$ solves the problem $\left(P_{\alpha}\right)$ posed on $B_{R_{*}}$ for some $\alpha \geq 0$. Since $f$ is of super-exponential type from Theorem 2.1 we obtain that $\alpha=0$. 


\section{Bifurcation analysis of the BRANCH CONVERGING TO A SINGULAR SOLUTION}

Let $f:[0, \infty) \rightarrow(0, \infty)$ (in particular, $f(0)>0$ ) be a $C^{3}$ nondecreasing convex nonlinearity which has one of the following forms for $m \in \mathbb{R}$ and all large $t>0$ :

(f1) $f(t)=t^{m} e^{t^{2}-t^{\mu}}, 1<\mu<2$,

(f2) $f(t)=t^{m} e^{t^{2}-t^{\mu}}, 0<\mu<1$ or $f(t)=t^{m} e^{t^{2}+t^{\mu}}, 0<\mu<2$.

Consider the following problem depending on a parameter $\lambda>0$ :

$$
\left\{\begin{aligned}
-\Delta u & =\lambda f(u) \\
u & >0 \\
u & =0 \text { on } \partial B_{1} .
\end{aligned} \text { in } B_{1},\right.
$$

Let $\mathcal{S}=\left\{(\lambda, u) \in \mathbb{R}^{+} \times C^{2, \gamma}(\bar{\Omega}) \mid u\right.$ solves $\left.\left(P_{\lambda}\right)\right\}$ denote the set of solutions of $\left(P_{\lambda}\right)$. Using tools from bifurcation theory and Theorem 3.2 we describe qualitative properties of a branch of solutions to the problem $\left(P_{\lambda}\right)$ with the above choice of $f$. In particular, we highlight the fact that we obtain at least two solutions to $\left(P_{\lambda}\right)$ when $f$ is of the form $(f 1)$ for certain small ranges of $\lambda$ (see property (3) in Theorem 4.1 below).

Theorem 4.1. Let $f$ be of the form $(f 1)$ or $(f 2)$. Then there exists a connected branch of solutions $\mathcal{C}$ in $\mathcal{S}$ and a positive real number $\Lambda$ with the following properties:

(1) $\mathcal{C} \subset(0, \Lambda] \times C^{2, \gamma}(\bar{\Omega})$ for some $0<\gamma<1$.

(2) For $0 \leq \lambda \leq \Lambda,\left(\lambda, w_{\lambda}\right) \in \mathcal{C}$, where $w_{\lambda}$ is the minimal solution to $\left(P_{\lambda}\right)$.

(3) (Bending) $\exists \delta>0$ such that for $\lambda \in(\Lambda-\delta, \Lambda)$, there exists another solution $u_{\lambda}$ with $\left(\lambda, u_{\lambda}\right) \in \mathcal{C}$. If $f$ is of the form $(f 2)$, in fact we can choose $\delta=\Lambda$.

(4) If $f$ is of the form $(f 1), \exists \epsilon>0$ such that $\left(\lambda, u_{\lambda}\right) \in \mathcal{C}, \lambda \leq \epsilon \Rightarrow u_{\lambda}=w_{\lambda}$.

(5) (Convergence to singular solution) If $f$ is of the form $(f 1)$, there exists a pair $\left(\lambda^{*}, u^{*}\right)$ with $0<\lambda^{*} \leq \Lambda, u^{*}$ a singular solution to $\left(P_{\lambda^{*}}\right)$ and a sequence $\left\{\left(\lambda_{n}, u_{n}\right)\right\} \subset \mathcal{C}$ such that $\lambda_{n} \rightarrow \lambda^{*}, u_{n}(0) \rightarrow \infty$ and $u_{n} \rightarrow u^{*}$ in $C_{l o c}^{2}\left(B_{1} \backslash\{0\}\right)$.

(6) (Concentration) If $f$ is of the form $(f 2)$, there exists a sequence $\left\{\left(\lambda_{n}, u_{n}\right)\right\}$ $\subset \mathcal{C}$ such that $\lambda_{n} \rightarrow 0, u_{n}(0) \rightarrow \infty$ and $\left|\nabla u_{n}\right|^{2} d x \rightarrow 4 \pi \delta_{0}$ in the sense of measure.

Proof. From the Gidas-Ni-Nirenberg symmetry result, we see that all solutions of $\left(P_{\lambda}\right)$ are radially symmetric. The existence of the connected branch $\mathcal{C}$ follows from the Crandall-Rabinowitz Theorem (see [7]). First, observe that we can get the existence and the uniqueness of a branch of minimal solutions to $\left(P_{\lambda}\right)$ near $(0,0)$ using the Implicit Function Theorem (since $f(0)>0$ ). In fact, using sub- and supersolution techniques, we can extend this local branch to a maximal branch of minimal solutions $\left\{\left(\lambda, w_{\lambda}\right)\right\}$ for $\lambda \in(0, \Lambda)$. We can show easily that $0<\Lambda<\infty$ since $f$ is superlinear at infinity and also that there is no solution to $\left(P_{\lambda}\right)$ for $\lambda>\Lambda$. By elliptic regularity, we can show that $w_{\lambda}$ belongs to $C^{2, \gamma}(\bar{\Omega})$ for some $\gamma \in(0,1)$. This proves (1)-(2).

Moreover, since $f^{\prime}$ is a nondecreasing function and $w_{\lambda}$ is the minimal solution (thus, stable), $\lambda_{1}\left(-\Delta-\lambda f^{\prime}\left(w_{\lambda}\right)\right)>0$ for $0 \leq \lambda<\Lambda$ (which implies that the map $\lambda \rightarrow w_{\lambda}$ is $C^{2}$ and $w_{\lambda}$ is locally unique for $\lambda \in[0, \Lambda)$ ). It follows that for $\lambda \in(0, \Lambda)$,

$$
\int_{\Omega}\left|\nabla w_{\lambda}\right|^{2}-\lambda \int_{\Omega} f^{\prime}\left(w_{\lambda}\right) w_{\lambda}^{2} \geq 0
$$


From Vitali's Convergence Theorem, we get that there exists a weak solution $u_{\Lambda}$ to $\left(P_{\Lambda}\right)$ such that $w_{\lambda} \rightarrow u_{\Lambda}$ in $H_{0}^{1}(\Omega)$ as $\lambda \rightarrow \Lambda$. Then, from elliptic regularity and Schauder estimates, we get that $u_{\Lambda} \in C^{2, \gamma}(\bar{\Omega})$. From the above, it follows that

$$
\lambda_{1}\left(-\Delta-\Lambda f^{\prime}\left(u_{\Lambda}\right)\right)=0 .
$$

Using the Fredholm Alternative and letting $L=-\Delta-\Lambda f^{\prime}\left(u_{\Lambda}\right)$, it is easy to see that $C_{0}^{2, \gamma}(\Omega)=\mathrm{N}(L) \oplus \mathrm{R}(L)$, where $\mathrm{N}(L)$ (resp. $\mathrm{R}(L) \subset C^{0, \gamma}(\bar{\Omega})$ ) denotes the kernel of $L$ (resp. the range of $L$ ). From the Krein-Rutman Theorem, it follows that $\mathrm{N}(L)$ is one dimensional, spanned by a positive function $\phi_{1}$. Moreover, since $L$ is self-adjoint, $\mathrm{R}(L)=\left\{\phi_{1}\right\}^{\perp}$. Then the transversality condition is satisfied since

$$
-\int_{\Omega}\left(f^{\prime}\left(u_{\Lambda}\right)+\Lambda f^{\prime \prime}\left(u_{\Lambda}\right) \frac{d w_{\lambda}}{d \lambda}(\Lambda)\right) \phi_{1}^{2}<0 .
$$

Therefore, we can apply Theorem 1.7 in [7, and there exists $\nu>0$ such that the solutions to $\left(P_{\lambda}\right)$ near $\left(\Lambda, u_{\Lambda}\right)$ form a twice continuously differentiable curve $\mathcal{B}=\{(\lambda(s), \tilde{u}(s))|| s \mid<\nu\}$ with $\lambda(0)=\Lambda$, and from computation of Theorem 4.8 in [8] (see also Theorem 1.1 in [9]), $\lambda^{\prime}(0)=0, \lambda^{\prime \prime}(0)<0$. Therefore, the curve $\mathcal{C}$ bends to the left at $\lambda=\Lambda$. Appealing to the uniqueness and multiplicity result in [11] (see Theorems 1.2,1.3 and Proposition 8.3), we complete the proof of (3).

If $f$ is of the form (f1), from property (4) and the global bifurcation theory of Rabinowitz (see 14) we see that there exists $\left(\lambda_{n}, u_{n}\right) \in \mathcal{C}$ and $\lambda_{*}>0$ such that $\lambda_{n} \rightarrow \lambda_{*}$ and $u_{n}(0) \rightarrow \infty$ (since $\mathcal{C}$ cannot "cross" the minimal solutions branch which is locally unique). Making the preliminary reductions as in Section 7 in [1] and from Theorem 3.3, (5) follows.

If $f$ is of the form (f 2), from property (3) and the global bifurcation result of Crandall-Rabinowitz again, we get that $\lambda=0$ is the unique asymptotic bifurcation line for $\mathcal{C}$. Let $u_{\lambda}, \lambda \in(0, \lambda)$ be as in (3). Clearly, we have that $u_{\lambda}(0) \rightarrow \infty$ as $\lambda \rightarrow 0$. We obtain (6) by using Theorem B in [2].

Remark 4.2. We guess that if $f$ is of the form (f 1$), \mathcal{C}$ has infinitely many turning points similar to the problems studied in [10] and [12].

Remark 4.3. From properties (5) and (6) in Theorem 4.1, we get two different situations determined by the asymptotic behaviour of $f$. For the detailed microscopic blow-up analysis of $u_{\lambda}$ see [2] and [4], where more general cases are considered.

\section{ACKNOWLEDGEMENT}

One of the authors would like to thank Professor Adimurthi for helpful discussions on some of the material in this work.

\section{REFERENCES}

[1] Adimurthi, Existence of positive solutions of the semilinear Dirichlet problem with critical growth for the n-Laplacian, Ann. Sc. Norm. Sup. Pisa Cl. Sci. (4) 17 (1990), 393-413. MR.1079983 (91j:35016)

[2] Adimurthi and S. Prashanth, Failure of Palais-Smale condition and blow-up analysis for the critical exponent problem in $\mathbf{R}^{2}$, Proc. Indian Acad. Sci. Math. Sci. 107, No. 3 (1997), 283-317. MR 1467434 (98j:35056)

[3] Adimurthi and S. Prashanth, Critical exponent problem in $R^{2}$-border-line between existence and non-existence of positive solutions for Dirichlet problem, Advances Differential Equations 5, No. 1-3 (2000), 67-95. MR1734537(2000i:35054) 
[4] Adimurthi and M. Struwe, Global compactness properties of semilinear elliptic equations with critical exponential growth, J. Funct. Anal. 175, No. 1 (2000), 125-167. MR.1774854 (2001g:35063)

[5] H. Brézis and P.-L. Lions, A Note on Isolated Singularities for Linear Elliptic Equations, Mathematical Analysis and Applications, Part A, Advances in Math. Suppl. Stud., 7A, Academic Press, New York-London, 1981, 263-266. MR634242 (83e:35039)

[6] H. Brezis and F. Merle, Uniform estimates and blow-up behaviour for solutions of $-\Delta u=$ $V(x) e^{u}$ in two dimensions, Comm. Partial Differential Equations 16, No. 8-9 (1991), 12231253. MR.1132783 (92m:35084)

[7] M.G. Crandall and P.H. Rabinowitz, Bifurcation from simple eigenvalues, J. Funct. Anal. 8 (1971), 321-340. MR0288640 (44:5836)

[8] M.G. Crandall and P.H. Rabinowitz, Bifurcation, perturbation of simple eigenvalues and linearized stability, Arch. Ration. Mech. Anal. 52 (1973), 161-180. MR0341212 (49:5962)

[9] M.G. Crandall and P.H. Rabinowitz, Some continuation and variational methods for positive solutions of nonlinear elliptic eigenvalue problems, Arch. Ration. Mech. Anal. 58, No. 3 (1975), 207-218. MR0382848 (52:3730)

[10] E.N. Dancer, Infinitely many turning points for some supercritical problems, Ann. Mat. Pura Appl. (4) 178 (2000), 225-233. MR1849387 (2002g:35077)

[11] J. Giacomoni, S. Prashanth and K. Sreenadh, A global multiplicity result for $N$-Laplacian with critical nonlinearity of concave-convex type, J. Diff. Equations 232 (2007), 544-572. MR2286391 (2007k:35140)

[12] D.D. Joseph and T.S. Lundgren, Quasilinear Dirichlet problems driven by positive sources, Arch. Ration. Mech. Anal. 49 (1972/73), 241-269. MR0340701 (49:5452)

[13] T. Ogawa and T. Suzuki, Nonlinear elliptic equations with critical growth related to the Trudinger inequality, Asymptotic Analysis 12 (1996), 25-40. MR1373480 (98f:35054)

[14] P.H. Rabinowitz, Some global results for nonlinear eigenvalue problems, J. Funct. Anal. 7 (1971), 487-513. MR0301587 (46:745)

Tata Institute of Fundamental Research, Center for Applicable Mathematics, P.B. No. 6503, Sharadanagar, Chikkabommasandra, Bangalore 560065, India

E-mail address: dhanya@math.tifrbng.res.in

Laboratoire de Mathématiques Appliquées, Université de Pau et des Pays de l'Adour, B.P. 576, 64012 Pau cedex, France

E-mail address: jgiacomo@univ-pau.fr

Tata Institute of Fundamental Research, Center for Applicable Mathematics, P.B. No. 6503, Sharadanagar, Chikkabommasandra, Bangalore 560065, India

E-mail address: pras@math.tifrbng.res.in 